\title{
Desafios e Experiências no Ensino de Programação Java através de Educação a Distância para Pessoas com Deficiência
}

\author{
Flavio Alves dos Santos ${ }^{1}$, Luiz Alexandre Castelo Branco Gomes ${ }^{1}$, \\ Eudenia Magalhães Barros ${ }^{2}$, Francisco Tearle de Oliveira Pinheiro ${ }^{3}$, \\ Lidiane Castro Silva ${ }^{1}$, Marcos Devaner do Nascimento ${ }^{1}$, \\ Paulo Henrique Mendes Maia ${ }^{1}$ \\ ${ }^{1}$ Universidade Estadual do Ceará - UECE \\ ${ }^{2}$ Universidade Federal do Ceará - UFC \\ ${ }^{3}$ Instituto Federal de Educação, Ciência e Tecnologia do Ceará - IFCE \\ \{flavio, luiz.alexandre, eudenia, tearle, \\ lidcastro, devaner, paulo.henrique\}@dellead.com
}

\begin{abstract}
The IT Market is always demanding qualified professionals. Therefore, learning how to program is an essential requirement to get a good job. However, disabled people (DP) face several barriers when trying to learn programming, ranging from lack of technical knowledge to comprehension of the used language. This paper describes the challenges found during the application of three courses about notions of programming logic and Java development to DP using a distance education environment, beyond the solutions adopted to minimize the course evasion and to maximize the student's learning experience.
\end{abstract}

Resumo. O mercado de TI está sempre demandando profissionais qualificados. Portanto, aprender a programar (bem) é requisito essencial para conseguir uma vaga de trabalho. Entretanto, pessoas com deficiência (PcD) encontram diversas barreiras quando tentam aprender a programar, indo desde falta de domínio técnico até compreensão da linguagem utilizada. Este artigo descreve os desafios encontrados durante a aplicação de três cursos sobre noções de lógica e desenvolvimento com Java para PcD através de um ambiente de Educação a Distância (EaD), bem como discute as soluções pedagógicas e tecnológicas adotadas para minimizar a evasão dos cursos e maximizar o aprendizado dos alunos.

\section{Introdução}

No Brasil, existe cerca de 45 milhões de pessoas com deficiência (PcD), segundo o último Censo Demográfico [IBGE 2010]. Essa parcela da população encontra, historicamente, barreiras na obtenção de formação profissional pois, além de sofrer preconceito, há também dificuldades que instituições de ensino e capacitação profissional enfrentam para formar esses indivíduos e inseri-los no mercado de trabalho, como a necessidade de intérpretes e tradutores de Libras (Língua Brasileira de Sinais) para deficientes auditivos, infraestrutura e capacitação de equipe pedagógica e professores para atuar junto às especificidades da educação inclusiva. 
VI Congresso Brasileiro de Informática na Educação (CBIE 2017)

Anais do XXIII Workshop de Informática na Escola (WIE 2017)

Uma forma de tentar melhorar o processo de aprendizagem de PcD é através do uso de recursos tecnológicos, como softwares educativos, objetos de aprendizagem e tecnologias assistivas [Rocha et al. 2016, Fernandes et al. 2016]. Soares [2014] relata a necessidade de oferecer soluções tecnológicas capazes de qualificar um grande número de $\mathrm{PcD}$, explorando de maneira adequada suas habilidades e potencializando suas competências.

Dentre essas soluções, a Educação a Distância $(\mathrm{EaD})$ exerce papel fundamental na inclusão digital de PcD na medida em que pode complementar - e, muitas vezes, melhorar - o conteúdo didático e contornar os problemas do ensino presencial. Para tanto, ambientes virtuais de aprendizagem, como Moodle, Teleduc, e OpenOlat vêm sendo usados na oferta de curso para PcD [Araujo et al. 2016]. Através da EaD, é possível qualificar PcD para exercerem os mais diversos cargos em diferentes atividades, e não apenas aproveitálos em trabalhos subalternos, ou que exigem baixo nível técnico.

Apesar dos avanços na oferta de cursos a distância para $\mathrm{PcD}$, ainda há problemas na capacitação de profissionais para a área de Tecnologia da Informação (TI). Particularmente, o mercado de desenvolvimento de software cresce significativamente no Brasil e apresenta grandes oportunidades para jovens programadores. Contudo, poucas iniciativas têm sido propostas para a qualificação de PcD para o mercado de TI.

Com o intuito de preencher essa lacuna, o Laboratório de Educação a Distância para Pessoas com Deficiência (LEAD), em parceria com a Universidade Estadual do Ceará (UECE), pesquisa e desenvolve soluções de EaD acessíveis a pessoas com deficiência. Recentemente, foi ofertado um curso à distância de desenvolvimento Java, composto de três módulos principais (Lógica de Programação, Java Básico e Java Intermediário), que ao todo somaram 370 horas, através de uma plataforma web com recursos de acessibilidade.

Este artigo apresenta os desafios encontrados no processo de aplicação desse curso, levando em conta as peculiaridades envolvidas, como a diversidade entre as pessoas com deficiência (física, auditiva e baixa visão) e ser totalmente a distância (i.e., sem encontros presenciais). O artigo está dividido da seguinte forma: a seção 2 apresenta conceitos que embasam o trabalho apresentado; a seção 3 discute os principais trabalhos relacionados; a seção 4 descreve a metodologia aplicada nos cursos, enquanto os desafios e soluções para os problemas encontrados são detalhados na seção 5; por fim, a seção 6 traz as conclusões e trabalhos futuros.

\section{Fundamentação Teórica}

Em um país de grandes dimensões como o Brasil, o acesso ao conhecimento ainda é muito precário [Gadotti 1997]. Essa situação se agrava ainda mais quando se trata de educação para PcD. Com o intuito de democratizar o ensino e apresentar uma alternativa para amenizar esse imenso problema existente no país, surge a EaD com a perspectiva de ofertar educação para parte da população não atendida pelo sistema tradicional de ensino.

Apesar de a EaD poder contribuir com a formação de $\mathrm{PcD}$, faz-se necessária a adaptação dos recursos tecnológicos utilizados nos cursos a distância a fim de que se tornem acessíveis para esse público. Dentre os recursos, destacam-se os Ambientes Virtuais de Aprendizagem (AVA), espaços que têm como objetivos promover a interação entre as 
pessoas e os conteúdos, integrar as mais diversas mídias, aproximar professores e alunos, e permitir fácil gerenciamento dos dados, dentre outros [Barbosa 2005]. Diante disso, surge a necessidade de pesquisar e produzir materiais didáticos digitais que utilizem tecnologias assistivas e promovam o aprendizado através dos recursos de acessibilidade e adaptabilidade voltados para pessoas com deficiência.

A acessibilidade em materiais digitais, segundo Dias [2003], significa que qualquer pessoa, usando qualquer tipo de tecnologia virtual (gráficos, textuais, especiais para cegos ou para sistemas de computação móvel) é capaz de visitar e interagir com esses conteúdos, compreendendo claramente as informações neles apresentadas.

Nesse paradigma de educação inclusiva, os recursos de tecnologia de informação e comunicação utilizados no processo de ensino e aprendizagem precisam também ser acessíveis. Incluem-se, nesse caso, os objetos de aprendizagem (OAs), definidos como uma entidade, digital ou não, que pode ser usada e reutilizada ou referenciada durante um processo de suporte tecnológico ao ensino e aprendizagem [IEEE 2017]. Uma característica importante no uso de OAs por pessoas com deficiência é que não há uma barreira rígida de tempo e espaço. A aprendizagem pode ocorrer de acordo com o ritmo de cada um, podendo, inclusive, realizar uma ou mais atividades quantas vezes quiser ou forem necessárias.

\section{Trabalhos Relacionados}

Em prol de aumentar a eficácia do ensino para $\mathrm{PcD}$, as tecnologias da informação vão ajudar com a construção de inúmeras ferramentas de apoio aos mais diferentes tipos de indivíduos. Boscarioli et al. [2015] relatam o acompanhamento de um aluno surdo durante dois anos no curso de Ciência da Computação, onde puderam observar que a falta de conhecimento das línguas nativas, seja para a pessoa com deficiência auditiva (usuária de Libras), seja para o professor (usuário da Língua Portuguesa), é uma das principais dificuldades. Neste estudo, um dos professores iniciou um curso de Libras, não para se tornar fluente na língua, mas para perceber a velocidade com a qual o intérprete traduz a informação passada. Esse desafio encontrado no ensino presencial pode ser atenuado com o ensino a distância, já que o conteúdo estará sempre disponível em vídeo para ser revisto sempre que o aluno desejar. Embora o estudo pareça adequado, seus resultados mostraram que a aferição da aprendizagem do aluno não pode ser determinada, pois o trabalho de passar o conteúdo ao aluno depende do grau de instrução do intérprete em relação ao conteúdo que se quer passar.

Com isso, surgem ferramentas para o ensino de Libras, como em Rocha et al. [2016] , no qual os autores utilizam elementos de gamificação para desenvolver um aplicativo para ensino da língua de sinais, de forma mais didática e lúdica. Reinoso, Almeida e Tavares [2016] desenvolveram uma plataforma para Construção de Arquitetura Pedagógica (CAP) para ensinar Libras na formação de intérpretes, permitindo ao instrutor desenvolver atividades coletivas e individuais. Estes dois trabalhos mostram uma perspectiva muito relevante, o que se espera de um estudo baseado fortemente na literatura. Porém, a validação em produção das duas ferramentas pode divergir dos resultados em dos testes.

Os deficientes visuais também podem ser contemplados com a tecnologia através do desenvolvimento de meios que os ajudam, principalmente, no uso do computa- 
VI Congresso Brasileiro de Informática na Educação (CBIE 2017)

Anais do XXIII Workshop de Informática na Escola (WIE 2017)

dor como, por exemplo, os leitores de telas como o NVDA [NV Access 2017] e JAWS [Freedom Scientific 2017]. Luque, Brandão e Brandão [2016] desenvolveram o Model2gether, uma ferramenta gratuita para o ensino de modelos aos cegos, considerando tanto acessibilidade quanto mecanismos de cooperação. Assim como os trabalhos de Rocha et al. [2016] e Reinoso, Almeida e Tavares [2016], o Model2Gether apresentado por Luque, Brandão e Brandão [2016] não possui validação em massa. É uma ferramenta que se mostra muito relevante e necessária, que mostra uma abordagem bem concisa no ensino de temas da computação para pessoas com deficiência visual, usuários de leitores de tela, por exemplo.

\section{O curso Desenvolvimento de Software em Java para PcD e sua Metodologia}

O curso Desenvolvimento de Software em Java é uma qualificação na área de desenvolvimento de software ofertada através de uma plataforma web proprietária elaborada para dispor, de forma eficiente, de recursos acessíveis que permitam a inclusão de $\mathrm{PcD}$, particularmente pessoas com surdez, pessoas com mobilidade reduzida e pessoas com baixa visão. O curso consisti-se em três módulos: (i) Lógica de Programação (70h/a), no qual eram ensinados conceitos básicos para o desenvolvimento do raciocínio para a resolução de problemas através da lógica; (ii) Java Básico (150h/a), onde eram ensinados conceitos iniciais da linguagem Java e suas teorias, como orientação a objetos, tipos de dados, tratamento de exceções e interfaces gráficas; (iii) Java Intermediário (150h/a), abrangendo conceitos avançados de uso de tipos de dados, UML, programação para redes, banco dados e introdução à programação para a web.

Devido à aplicação do curso ter sido promovida junto ao Governo do Estado do Ceará, através de uma de suas políticas de inclusão social, o público-alvo deveria ser formado, prioritariamente, por pessoas com deficiência auditiva, física e baixa visão, e pessoas que se encontravam em situação de vulnerabilidade social cuja renda familiar fosse de até 1 salário mínimo, residentes em Fortaleza, ou na área metropolitana. A inscrição no curso era gratuita e os alunos selecionados tinham que atender ao perfil acima descrito.

Dado o perfil exigido, muitos alunos selecionados não possuíam conhecimento em assuntos que eram necessários para o entendimento do curso. Portanto, foi realizado um módulo inicial de nivelamento, com carga horária de 60h/a, aplicado presencialmente, com o objetivo de proporcionar recuperação e aprimoramento de conhecimentos básicos em Informática, Lógica Matemática e Inglês Instrumental, tentando assim reduzir o índice de reprovação e evasão. Os professores dessa fase de nivelamento eram graduandos em Ciência da Computação e Licenciatura em Letras e Matemática.

Cada módulo a distância possuiu um conjunto de webaulas instrutivas em texto, complementadas com as videoaulas, que abordavam temas específicos. No que tange às atividades do curso, foram utilizados diferentes objetos de aprendizagem, como fóruns de discussão, oficinas práticas de programação e questionários de assimilação de conteúdo. Para concluir, ao final de cada aula, o aluno era submetido a uma avaliação online que agregava todos os conteúdos estudados. Essa avaliação, juntamente com o fórum e a oficina, constituíram a média e frequência final dos alunos.

Os módulos eram condicionados e cada aluno só poderia avançar no curso caso 
VI Congresso Brasileiro de Informática na Educação (CBIE 2017)

Anais do XXIII Workshop de Informática na Escola (WIE 2017)

cumprisse, com êxito, as tarefas propostas, alcançando média igual ou superior à 6,0. Cada módulo tinha um conjunto de tutores para acompanhar as atividades e ajudar os alunos em suas dúvidas.

\section{Desafios e Soluções}

A EaD surgiu como um meio de levar educação para as pessoas nos mais remotos locais do mundo, facilitando o acesso ao ensino de pessoas mais abastadas [Hermida and BONFIM 2006]. Com a evolução da TI, tornou-se possível a criação de novas ferramentas que ajudassem $\mathrm{PcD}$ a ter acesso à informação e à educação. No entanto, a formação através da $\mathrm{EaD}$ é, em suma, repleta de muitos desafios. A dificuldade de acesso ao conteúdo, a qualificação e a evasão são os maiores destes desafios.

A seguir são discutidos os principais problemas encontrados durante a aplicação do curso e as soluções propostas, dividindo-os em duas categorias: aspectos pedagógicos, que abordam situações vividas no contexto da educacional do curso, e aspectos tecnológicos, que descrevem ferramentas e objetos de aprendizagem desenvolvidas.

\subsection{Aspectos Pedagógicos}

A equipe pedagógica do LEAD foi responsável pelo apoio e acompanhamento didático e pedagógico dos alunos que atuaram nos cursos ofertados. Este grupo foi composto por um coordenador professor do curso de Ciência da Computação, dois pedagogos e uma socióloga, responsáveis pelo acompanhamento dos cursos.

Um dos grandes desafios dessa equipe foi apresentar a educação a distância para os alunos, pois a maior parte deles nunca havia estudado nessa modalidade. A conscientização sobre os direitos e deveres dos alunos foi bastante trabalhada com eles no início do curso.

Uma importante função da equipe foi tratar sobre as questões pedagógicas das disciplinas, incluindo apoio aos tutores em relação ao acompanhamento dos alunos, bem como suas participações na plataforma. Para que essas ações realmente fossem efetivas, foram realizadas reuniões semanais com os tutores para sanar possíveis dúvidas. Tais reuniões tiveram também um caráter formativo, discutindo assuntos relacionados a EaD e Acessibilidade, nas quais os tutores trocavam experiências e discutiam os problemas em comum, promovendo maior união e comprometimento com as atividades.

A partir dessas reuniões, eram gerados relatórios com índices da participação dos alunos e, com base nesses dados, a equipe entrava em contato tanto com os alunos através de e-mails, telefonemas e ferramentas disponíveis na plataforma para felicitar aqueles que estavam acompanhando periodicamente as aulas, de modo a incentivá-los a continuar, bem como notificar os alunos que não estavam participando das aulas, indagando-os sobre os motivos da sua não participação. Depois de detectado algum tipo de problema que estivesse afastando os alunos eram criadas estratégias para fazer com que esses alunos retornassem às aulas.

A principal tarefa da equipe pedagógica foi pensar a modalidade de $\mathrm{EaD}$, organizando os aspectos metodológicos utilizados nas atividades e também as tecnologias implantadas na plataforma, com o objetivo principal de facilitar o aprendizado dos alunos. 
VI Congresso Brasileiro de Informática na Educação (CBIE 2017)

Anais do XXIII Workshop de Informática na Escola (WIE 2017)

\subsection{Aspectos tecnológicos}

O projeto de interface de todo o ambiente de aprendizado incorpora as principais ferramentas de acessibilidade já existentes, como alto contraste, comando de voz, vídeos em Libras, alertas luminosos, adaptação do layout do menu que facilite a navegação, ergonomia dos botões de acesso, dentre outros. Mesmo assim, durante a aplicação dos cursos, percebeu-se a dificuldade que os alunos, de forma geral, tinham para utilizar as ferramentas de desenvolvimento de código, em especial os alunos surdos. Além disso, a comunicação entre alunos surdos usuários da Libras e o tutor mostrava-se ineficiente, pois a língua de sinais apresentava-se como um desafio para o tutor, assim como o português para o aluno.

Dadas as dificuldades encontradas, pensou-se em ferramentas tecnológicas que pudessem auxiliar PcD no seu processo de aprendizado e comunicação. Dentre as soluções desenvolvidas, destacam-se: (i) JLOAD, uma ferramenta acessível para atividades práticas de programação; (ii) JAD, um depurador de código visual, com lançamentos de exceções em Libras; (iii) perfil de intérprete na plataforma web, pois assim o intérprete de Libras atende as solicitações de tradução de fóruns e mensagens, e; (iv) a Fábrica de Sinais, que disponibiliza um conjunto de sinais em Libras para termos próprios do contexto de programação, como classe, exceção, herança, entre outros. A seguir, serão descritos, em mais detalhes, esses quatro objetos de aprendizagem.

\subsubsection{JLOAD}

O JLOAD (Java Learning Object to Assist the Deaf) [Silva et al. 2014] é um objeto de aprendizado criado com base nas teorias do aprendizado ativo e zona de desenvolvimento proximal (ZDP). Ele possui um IDE simples e ferramentas de colaboração para a assistência e um acompanhamento das atividades práticas de forma remota e assíncrona. Essa colaboração envolve alunos, tutores e intérpretes de Libras.

Uma atividade no JLOAD requer alguma codificação para ser compilada e executada e tem seu próprio conjunto de instruções (exibido em Libras e Português). O aluno pode enviar o código-fonte, solicitar e receber ajuda de um tutor, tudo feito dentro do ambiente do JLOAD. Essa abordagem tudo-em-um possibilita ao aluno um ambiente integrado de aprendizado e codificação, trazendo maior mobilidade, pois tudo está em um ambiente web. A interface do JLOAD e os recursos disponibilizados nele podem ser visto na Figura 1.

\subsubsection{JAD}

Durante a aplicação dos módulos, foi possível perceber a dificuldade que alunos surdos tinham para desenvolver e evoluir seus códigos. Decidiu-se, então, analisar o desempenho de alunos surdos e ouvintes durante a tarefa de depuração de um código Java. Os resultados mostraram que programadores surdos têm mais dificuldade para desempenhar essa tarefa [Nascimento et al. 2014], sendo esta dificuldade atenuada quando utilizado um depurador visual [Nascimento et al. 2016]. Por esta razão, decidiu-se propor o JAD (Java Accessible Debugguer), um depurador visual acessível. O objetivo da ferramenta é 
VI Congresso Brasileiro de Informática na Educação (CBIE 2017)

Anais do XXIII Workshop de Informática na Escola (WIE 2017)

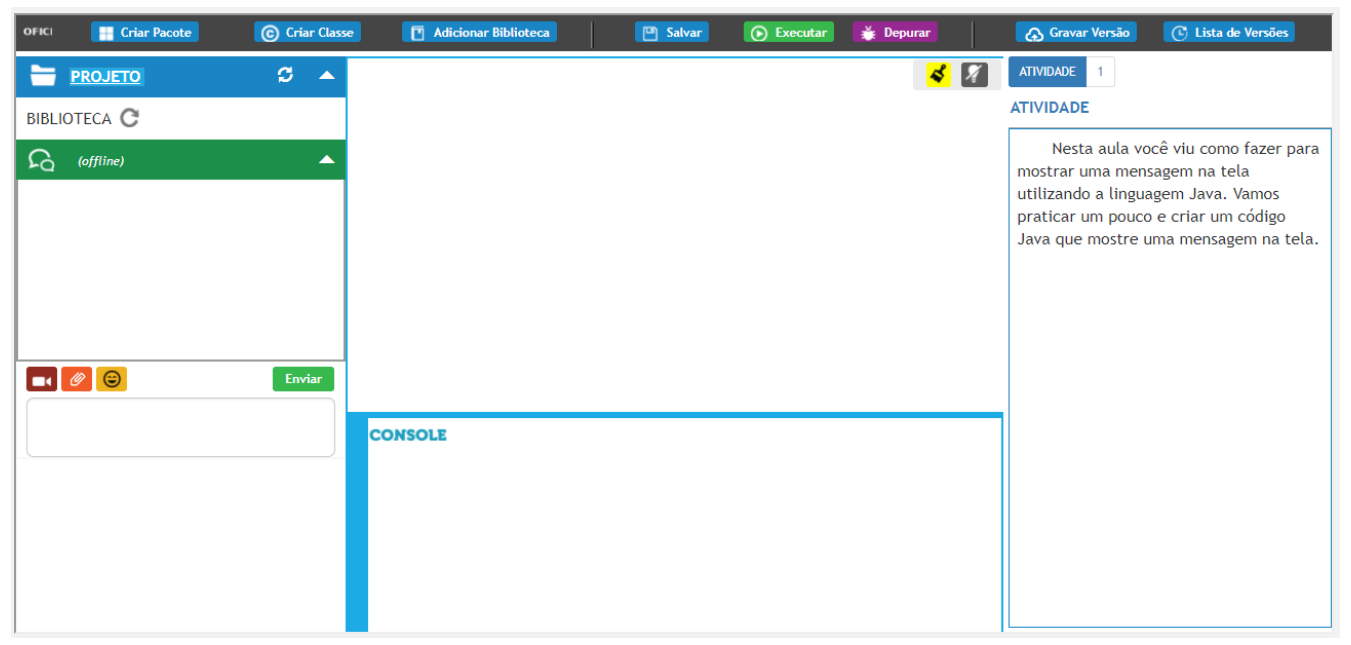

Figura 1. Interface do JLOAD <omitido para revisão>

reduzir o esforço cognitivo aplicado pela pessoa com surdez durante a depuração e compreensão de erros na linguagem Java, oferecendo uma ferramenta acessível que estimule o processo de aprendizagem e reduza os riscos de evasão.

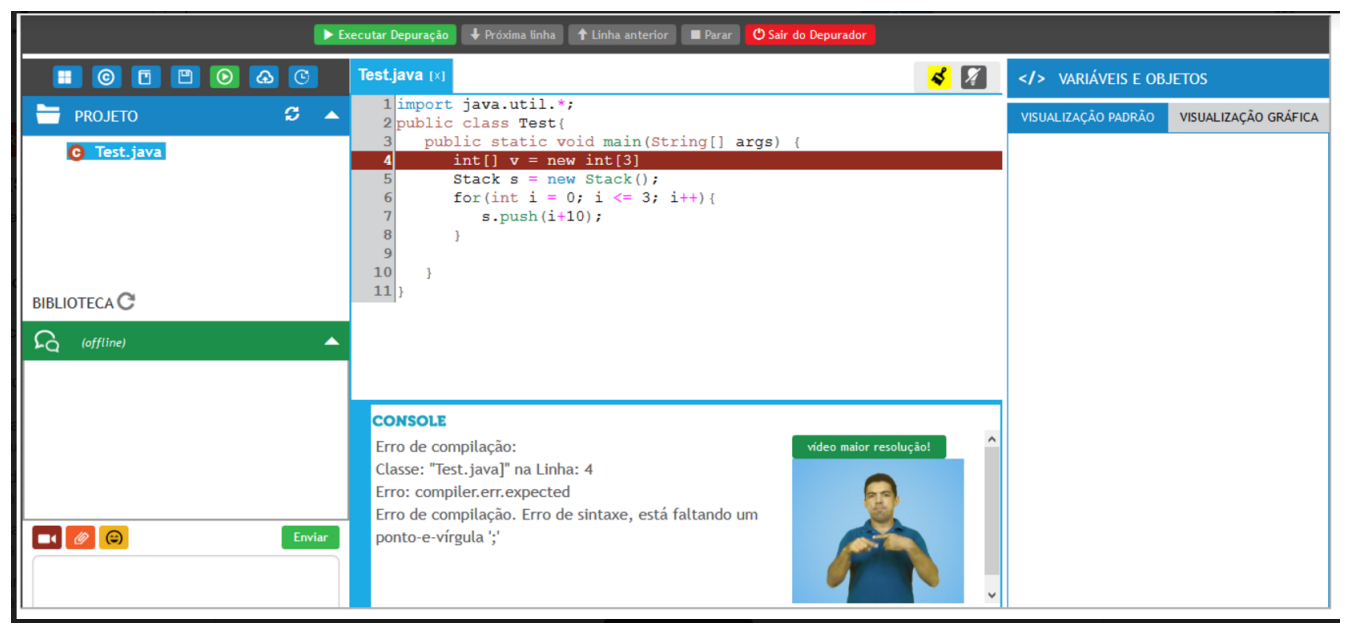

Figura 2. Interface do JAD <omitido para revisão>

\subsubsection{Perfil de Intérprete}

Adicionalmente, foi desenvolvido para a plataforma uma nova ferramenta para permitir a automatização do processo de tradução para Libras de textos publicados em Português por alunos ouvintes (não surdos) nas interações realizadas nos fóruns, por exemplo. Com a nova ferramenta, é possível que um aluno surdo solicite a tradução de algum texto em Português, quando um intérprete de Libras é notificado através da plataforma e publica o vídeo em Libras com a tradução correspondente para que todos vejam, alunos surdos e ouvintes. Esta inovação garante que todos os alunos surdos em um ambiente de ensino a distância tenham sempre uma abordagem bilíngue quando acharem necessário. 
VI Congresso Brasileiro de Informática na Educação (CBIE 2017)

Anais do XXIII Workshop de Informática na Escola (WIE 2017)

\subsubsection{Fábrica de Sinais}

A Fábrica de Sinais é uma ferramenta web que tem como objetivo permitir que alunos surdos participem, por meio de um ambiente colaborativo, da criação de novos sinais das palavras que ainda não tem tradução para Libras. Isso acontece por meio do envio de vídeos com sugestões de sinais e votação entre os participantes de uma discussão pelos próprios usuários da ferramenta.

De forma intuitiva, o usuário pode realizar o seu cadastro na ferramenta, onde o usuário deve enviar um vídeo ou gravá-lo através de uma webcam, explicando qual conceito ele considera importante ser criado um sinal em Libras. A partir disso, o criador desse item é redirecionado para a página de "Discussão", onde todos os usuários poderão enviar suas sugestões de sinais e votar nos que considerarem mais apropriados para representar aquela ideia. Nessa tela, todos podem ver os sinais e comentários inseridos, organizados tanto pelo maior número de votos quanto pelos mais recentemente enviados. Após criar uma discussão, ela fica organizada nas "categorias", onde outras discussões estão armazenadas, possibilitando que o usuário participe.

\section{Resultados}

Como resultado da implantação do programa de capacitação, 1.793 pessoas foram inscritas nos processos seletivos realizados. Destes, 1.036 alunos foram matriculados em 34 turmas criadas de abril de 2016 a março de 2017. Do total de alunos matriculados, 52\% foram aprovados de acordo com os critérios avaliativos publicados nos editais de seleção. Destes, $63 \%$ eram alunos em situação de vulnerabilidade social, e outros $15 \%$ composto por pessoas com deficiência. O índice geral de satisfação dos alunos foi de $91 \%$, onde foram avaliados os critérios de organização didático-pedagógica, corpo docente e tutorial, acessibilidade, entre outros, pelos próprios alunos.

Os resultados por módulos apresentam em detalhes como foi o desempenho dos alunos de modo geral, e também dos alunos com deficiência. Para o módulo de Lógica de Programação, foram formadas 16 turmas ao longo do ano. Nelas, 524 alunos foram matriculados, e $292(55,7 \%)$ obtiveram aprovação. Do total de alunos matriculados, 105 eram alunos $\mathrm{PcD}$, dos quais 29 (27,6\%) obtiveram êxito.

No módulo de Java Básico, foram matriculados 267 alunos, distribuídos em 9 turmas. Destes, 155 (58\%) foram aprovados e habilitados para cursarem o módulo seguinte. Nesse mesmo universo, 29 alunos eram PcD, sendo que 10 (34\%) obtiveram êxito. No módulo de Java Intermediário, quase um ano após o início da aplicação, 143 alunos foram distribuídos em 5 turmas. Destes, 79 (55\%) foram aprovados. Com relação aos PcD, nesse módulo estavam matriculados 8 , e 6 obtiveram aprovação.

Os alunos que alcançaram o módulo de Java Intermediário, necessariamente, estiveram matriculados entre as turmas 1 a 10 de Lógica de Programação, isto porque, enquanto as formações aconteciam e turmas passavam de um módulo a outro, novas vagas para o módulo de Lógica de Programação ficavam disponíveis, ou seja, alunos matriculados nas turmas de 11 a 14 atingiram apenas o módulo de Java Básico 8 e 9 e as últimas turmas de Lógica de Programação (15 e 16) finalizaram sua formação neste módulo. Essa informação é relavante para compreender que os resultados de PcD poderiam ter um alcance maior nos módulos de Java Básico e Intermediário, caso eles tivessem participado 
VI Congresso Brasileiro de Informática na Educação (CBIE 2017)

Anais do XXIII Workshop de Informática na Escola (WIE 2017)

destes módulos.

Os alunos participaram ativamente da avaliação da plataforma $\mathrm{EaD}$, sugerindo novos requisitos para a acessibilidade, para os conteúdos e para as ferramentas de ensino, dentre eles melhorias na usabilidade da interface da plataforma, na tradução para Libras e na performance.

\section{Conclusões}

Este artigo teve como objetivo descrever os desafios encontrados durante aplicação de um curso composto por módulos de Lógica de Programação, Java Básico e Intermediário, através de uma plataforma $\mathrm{EaD}$, com foco no aprendizado de $\mathrm{PcD}$, a fim de discutir metodologias educacionais e tecnológicas para facilitar o aprendizado desse público. Foram apresentados dados sobre a metodologia de aplicação do curso, ferramentas desenvolvidas para tornar os conteúdos de programação ainda mais acessíveis, e os índices de aprovação entre os alunos com e sem deficiência.

É possível dizer que a curva de aprendizado dos alunos pôde ser reduzida com a aplicação de técnicas pedagógicas e interferência no processo de ensino-aprendizagem com ferramentas que tornam o aluno mais próximo do professor/tutor, o que reduziu muito a evasão e, desta forma, as dúvidas presentes durante o aprendizado foram sanadas de forma mais eficiente, evitando que o aluno se isolasse e não pudesse expor suas dúvidas. De maneira mais específica, a aplicação do JLOAD e do JAD auxiliaram no processo de aprendizagem, evitando que o aluno se frustrasse logo no início deste processo, com a instalação do ambiente, o que pode ser um pouco complexo para alunos com um grau de conhecimento em informática menor. Com estas ferramentas, balanceou-se o nível de conhecimento de todos os alunos das turmas, diminuindo a evasão.

É importante destacar que a implementação de novos requisitos na plataforma web de EaD é feita progressiva e continuamente de forma a atender Diretrizes de Acessibilidade para Conteúdo Web (WCAG) 2.0, que abrangem um vasto conjunto de recomendações que têm como objetivo tornar o conteúdo mais acessível [W3C 2008]. O cumprimento destas diretrizes fará com que o conteúdo se torne acessível a um maior número de pessoas com deficiência, incluindo as pessoas com cegueira e baixa visão, surdez e baixa audição, dificuldades de aprendizagem, limitações cognitivas, limitações de movimentos, incapacidade de fala, fotossensibilidade, bem como as que tenham uma combinação destas limitações.

Sabendo dos desafios da adequação de um ambiente $\mathrm{EaD}$ para $\mathrm{PcD}$, é fundamental a realização de pesquisas e estudos relacionados aos demais tipos de requisitos que abranjam outras funcionalidades acessíveis, para que seja possível o desenvolvimento de novas ferramentas que fomentem métodos educacionais e tecnológicos que permitam, também, a inclusão de pessoas com deficiência intelectual e pessoas com deficiência física severa (paraplegia e tetraplegia).

\section{Referências}

Araujo, T. A. C. d., Oliveira, F. C. d. M. B., Alves, M. L., Soares, M. I. d. S., Matos, A. S. d., Freitas, A. T. d., and Silva, L. C. (2016). Android accessible m-learning application for people with hearing and physical disabilities. In International Conference on Human-Computer Interaction, pages 215-220. Springer. 
VI Congresso Brasileiro de Informática na Educação (CBIE 2017)

Anais do XXIII Workshop de Informática na Escola (WIE 2017)

Barbosa, R. M. (2005). Ambientes virtuais de aprendizagem. Artmed.

Boscarioli, C., Galante, G., Oyamada, M. S., Zara, R. A., and Villwock, R. (2015). Aluno surdo na ciência da computação: Discutindo os desafios da inclusão.

Cláudia, D. (2003). Usabilidade na web: Criando portais acessíveis. Rio de Janeiro.

Fernandes, F. G., Cardoso, A., and Lamounier, E. (2016). Adaptação de jogos sérios para crianças com deficiência física nos membros superiores. In Anais dos Workshops do Congresso Brasileiro de Informática na Educação, volume 5, page 598.

Freedom Scientific, I. (2017). Jaws screen reader - best in class.

Gadotti, M. (1997). História das ideias pedagógicas. Ática.

Hermida, J. F. and BONFIM, C. R. d. S. (2006). A educação à distância: história, concepções e perspectivas. Revista HISTEDBR On-line, Campinas, n. especial, pages $166-181$.

IBGE (2010). Censo Demográfico 2010. Rio de Janeiro: IBGE.

IEEE, L. T. S. C. L. (2017). The learning object metadata standard.

Luque, L., Brandão, L., and Brandão, A. (2016). Model2gether: uma ferramenta de apoio ao ensino e a aprendizagem de modelos por cegos. In Anais dos Workshops do Congresso Brasileiro de Informática na Educação, volume 5, page 257.

Nascimento, M. D. d., Oliveira, F. C. d. M. B., and Freitas, A. T. d. (2014). How do deaf or hearing impaired programmers perform in debugging java code? In Brazilian Symposium on Computers in Education (Simpósio Brasileiro de Informática na Educação-SBIE), volume 25, page 593.

Nascimento, M. D. d., Oliveira, F. C. d. M. B., Freitas, A. T. d., and Silva, L. C. (2016). Visual debuggers and deaf programmers. In International Conference on Universal Access in Human-Computer Interaction, pages 26-37. Springer.

NV Access (2017). NVDA - NonVisual Desktop Access.

Reinoso, L., Almeida, R., and Tavares, O. (2016). Uma plataforma para construção de arquiteturas pedagógicas para a aprendizagem de libras. In Anais dos Workshops do Congresso Brasileiro de Informática na Educação, volume 5, page 531.

Rocha, P. R., Lima, R., Macedo, R., Maia, C., and Neto, F. M. (2016). Gamificação: Um aplicativo para o ensino da língua brasileira de sinais. In Anais dos Workshops do Congresso Brasileiro de Informática na Educação, volume 5, page 896.

Silva, L. C., de MB Oliveira, F. C., de Oliveira, A. C., and de Freitas, A. T. (2014). Introducing the jload: A java learning object to assist the deaf. In Advanced Learning Technologies (ICALT), 2014 IEEE 14th International Conference on, pages 579-583. IEEE.

Soares, M. I. d. S., Furtado Júnior, C. G., Silva, L. C., Oliveira, R. B. d., Lima, N. A. S., Soares, É. F., et al. (2014). Visual jo2: Um objeto de aprendizagem para o ensino de programação java a deficientes físicos e auditivos através do estímulo visual-um estudo de caso. RENOTE, 12(2).

W3C, W. W. W. C. (2008). Web content accessibility guidelines (WCAG) 2.0. 